\title{
Biologics in Pediatric Psoriasis and Atopic Dermatitis: Revolutionizing the Treatment Landscape
}

\author{
Jennifer B. Scott, MD; Amy S. Paller, MD
}

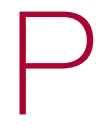

soriasis and atopic dermatitis (AD) can impact quality of life (QOL) in pediatric patients, warranting early recognition and treatment. ${ }^{1}$ Topical agents often are inadequate to treat moderate to severe disease, but the potential toxicity of systemic agents, which largely include immunosuppressives, limit their use in this population despite their effectiveness. Our expanding knowledge of the pathogenesis of psoriasis (tumor necrosis factor [TNF] $\alpha$ and IL-23/ $\mathrm{T}_{\mathrm{H}} 17$ pathways) and $\mathrm{AD}$ has led to targeted interventions, particularly monoclonal antibody biologics, which have revolutionized treatment for affected adults and more recently children. Several agents are approved by the US Food and Drug Administration (FDA) for pediatric psoriasis, and dupilumab is approved for pediatric AD. Herein, we discuss the latest developments in the treatment landscape for pediatric psoriasis and AD.

\section{Pediatric Psoriasis}

Methotrexate (MTX) and cyclosporine have been FDA approved for psoriasis in adults since 1972 and 1997, respectively. ${ }^{2}$ Before biologics, MTX was the primary systemic agent used to treat pediatric psoriasis, given its lower toxicity vs cyclosporine. The TNF- $\alpha$ inhibitor etanercept became the first FDA-approved biologic for pediatric psoriasis in 2016. Adalimumab has been available in Europe for children since 2015 but is not FDA approved. Certolizumab, a pegylated TNF- $\alpha$ inhibitor that distinctly fails to cross the placental barrier currently is in clinical trials (ClinicalTrials.gov identifier NCT04123795). Tumor necrosis factor $\alpha$ inhibitors have shown more rapid onset and greater efficacy during the first 16 weeks of use than MTX, including a head-to-head trial comparing MTX to adalimumab. ${ }^{3}$ A recent real-world study showed that pediatric patients receiving biologics, primarily TNF- $\alpha$ inhibitors, were more likely to achieve psoriasis area and severity index (PASI) 75 or clear/almost clear status (similar to PASI 90) than MTX and had higher drug survival rates. ${ }^{4}$

Ustekinumab targets both IL-12 and IL-23, which share the IL-23 receptor p40 subunit. It was the first biologic to target IL-23, which promotes the proliferation and survival of helper $\mathrm{T}$ cells $\left(\mathrm{T}_{\mathrm{H}} 17\right)$. Ustekinumab has led to greater reductions in PASI scores than TNF- $\alpha$ inhibitors. ${ }^{5,6}$ Pediatric trials of guselkumab, risankizumab, and tildrakizumab, all targeting the IL-23 receptor-specific p19 subunit, are completed or currently recruiting (NCT03451851, NCT03997786, NCT04435600). Ixekizumab is the first IL-17A-targeting biologic approved for children. ${ }^{7}$ Secukinumab and the IL-17 receptor inhibitor brodalumab are in pediatric trials (NCT03668613, NCT04305327, NCT03240809). One potential issue with $T_{\mathrm{H}} 17$ pathway inhibitors is their association with

From the Department of Dermatology, Northwestern University Feinberg School of Medicine, Chicago, Illinois. Dr. Paller also is from the Department of Pediatrics.

Dr. Scott reports no conflict of interest. Dr. Paller has been an investigator and/or consultant for AbbVie Inc; Abeona Therapeutics; Almirall; AnaptysBio; Asana; Boehringer Ingelheim; BridgeBio Pharma; Dermavant Sciences Ltd; Dermira, Inc; Eli Lilly and Company; Exicure; Forte Biosciences Inc; Galderma Laboratories, LP; Incyte Corporation; Janssen Pharmaceuticals, Inc; LEO Pharma; Lifemax Laboratories, Inc; Novartis; Pfizer; RAPT Therapeutics, Inc; Regeneron Pharmaceuticals; Sanofi Genzyme; Sol-Gel Technologies Ltd; and UCB.

The eTable is available in the Appendix online at www.mdedge.com/dermatology.

Correspondence: Amy S. Paller, MD, Department of Dermatology, 676 N St Clair St, Ste 1600, Chicago, IL 60611 (apaller@northwestern.edu). doi:10.12788/cutis.0110 
inflammatory bowel disease, a contraindication when considering if a patient is a potential candidate for treatment.

Skin disease can profoundly affect QOL during childhood and adolescence, a critical time for psychosocial development. In psoriasis, improvement in QOL is proportional to clearance and is greater when PASI 90 is achieved vs PASI $75 .{ }^{8}$ The high efficacy of IL-23 and IL-17A pathway inhibitors now makes achieving at least PASI 90 the new standard, which can be reached in most patients.

\section{Pediatric AD}

For $\mathrm{AD}$ in the pediatric population, systemic treatments primarily include corticosteroids, mycophenolate mofetil, azathioprine, cyclosporine, and MTX. Although cyclosporine was the favored systemic agent among pediatric dermatologists in one study, ${ }^{9}$ claims data analyses show that systemic corticosteroids are used much more often overall, prescribed in $24.4 \%$ (116,635 total cases) of children with $\mathrm{AD}$ vs nonsteroidal immunosuppressants in less than $0.5 \% .{ }^{10}$ Systemic steroids are impractical given their side effects and risk for disease rebound; however, no immunosuppressants are safe for long-term use, and all require frequent laboratory monitoring. The development of biologics for $\mathrm{AD}$ largely involves targeting $\mathrm{T}_{\mathrm{H}} 2$-driven inflammation. ${ }^{11}$ Dupilumab is the only FDA-approved biologic for moderate to severe pediatric $A D$, including in patients as young as 6 years of age. Dupilumab inhibits activation of the IL-4R $\alpha$ subunit, thereby blocking responses to its ligands, IL-4 and IL-13. Phase 3 trials are now underway in children aged 6 months to 5 years (NCT02612454, NCT03346434). The concomitant ameliorative effects of dupilumab on asthma and other allergic disorders, occurring in approximately $90 \%$ of children with moderate to severe AD, is an added benefit. ${ }^{12}$ Although dupilumab does not appear to modify the disease course in children with $\mathrm{AD}$, the possibility that early introduction could reduce the risk for later developing allergic disease is intriguing.

Adolescent trials have been started for lebrikizumab (NCT04392154) and have been completed for tralokinumab (NCT03160885). Both agents selectively target IL-13 to block $\mathrm{T}_{\mathrm{H}} 2$ pathway inflammation. The only reported adverse effects of IL-4R $\alpha$ and IL-13 inhibitors have been injection-site pain/reactions and increased conjunctivitis. ${ }^{13}$

The only other biologic for AD currently in clinical trials for adolescents is nemolizumab, targeting the receptor for IL-31, a predominantly $\mathrm{T}_{\mathrm{H}} 2$ cytokine that causes pruritus (NCT03989349). In adults, nemolizumab has shown rapid and potent suppression of itch (but not inflammation) without adding topical corticosteroids. ${ }^{14}$

\section{Advantages of Biologics and Laboratory Monitoring}

By targeting specific cytokines, biologics have greater and more rapid efficacy, fewer side effects, fewer drug interactions, less frequent dosing, and less immunosuppression compared to other systemic agents. ${ }^{3,4,15,16}$

Recent pediatric-specific guidelines for psoriasis recommend baseline monitoring for tuberculosis for all biologics but yearly tuberculosis testing only for TNF- $\alpha$ inhibitors unless the individual patient is at increased risk. ${ }^{2}$ No tuberculosis testing is needed for dupilumab, and no other laboratory monitoring is recommended for any biologic in children unless warranted by risk. This difference in recommended monitoring suggests the safety of biologics and is advantageous in managing pediatric therapy.

\section{Unanswered Questions: Vaccines and Antidrug Antibodies}

Although administration of killed vaccines is considered safe with all approved biologics, questions remain about the safety of administering live yaccines while on biologics, a particularly pertinent issue in younger children treated with dupilumab and other biologics for AD. Another unanswered question is the potential reduction in clinical response and drug durability with intermittent use of biologics due to the potential development of neutralizing antidrug antibodies (ADAs). The ability to discontinue medication intermittently is desirable, both to determine the natural course of the underlying disease and give a holiday as tolerated. Newer biologics are thought to have lower immunogenicity and less frequent ADA development. ${ }^{17-19}$ Even with TNF- $\alpha$ inhibitors, the presence of anti-ADAs is not temporally related to response in children with psoriasis. ${ }^{20}$ Longterm outcomes of the use of biologics in adults have been reassuring, and safety profiles of biologics studied thus far appear to be similar in children. ${ }^{21,22}$ However, understanding the potential long-term effects from the use of newly approved and emerging biologics in the pediatric population will require decades of study to ensure safety, including nonrandomized studies and postmarketing reports from regulatory agencies.

\section{Cost Considerations}

Biologics are disease and QOL altering for children with moderate to severe psoriasis or $\mathrm{AD}$; however, access to biologics often is an obstacle for patients and practitioners. Biologics cost $\$ 30,000$ to $\$ 60,000$ annually, while conventional systemic treatments such as MTX, cyclosporine, and acitretin cost $\$ 100$ to $\$ 3000$ annually, raising the question of cost effectiveness. In 2016, the Institute for Clinical and Economic Review concluded that biologics for psoriasis had reasonably good value based on improved QOL and concluded in 2017 that dupilumab had a benefit that outweighed its cost. $^{23,24}$ Prior authorizations and multiple appeals have been necessary to obtain approval, especially in the pediatric population..$^{25}$ This difficulty highlights the need for programs to cover the cost of biologics for all children, as well as registries to further assess effectiveness and 
long-term safety, especially compared to traditional systemic agents.

\section{On the Horizon}

Clinical trials for other therapies for children and adolescents are ongoing. Details on recommended dosing, approval status, and efficacy in trials are provided in the eTable. Given their high efficacy in adults with psoriasis, IL-23-specific and $\mathrm{T}_{\mathrm{H}} 17$ pathway biologics likely are similarly efficacious and raise the bar for the expectation of achieving PASI 90 and PASI 100 responses. The long-term safety, durability of responses, and ability to modify disease, particularly when started early in life (eg, preadolescence) and early in the disease course, remains to be determined.

\section{REFERENCES}

1. Na CH, Chung J, Simpson EL. Quality of life and disease impact of atopic dermatitis and psoriasis on children and their families. Children (Basel). 2019;6:133

2. Menter A, Cordoro KM, Davis DMR, et al. Joint American Academy of Dermatology-National Psoriasis Foundation guidelines of care for the management and treatment of psoriasis in pediatric patients. J Am Acad Dermatol. 2020;82:161-201.

3. Papp K, Thaci D, Marcoux D, et al. Efficacy and safety of adalimumab every other week versus methotrexate once weekly in children and adolescents with severe chronic plaque psoriasis: a randomised double-blind, phase 3 trial. Lancet. 2017;390:40-49.

4. Bronckers I, Paller AS, West DP, et al. A comparison of psoriasis severity in pediatric patients treated with methotrexate vs biologic agents. JAMA Dermatol. 2020;156:384-392.

5. Landells I, Marano C, Hsu MC, et al. Ustekinumab in adolescent patients age 12 to 17 years with moderate-to-severe plaque psoriasis: results of the randomized phase 3 CADMUS study. J Am Acad Dermatol. 2015;73:594-603.

6. Philipp S, Menter A, Nikkels AF, et al. Ustekinumab for the treatment of moderate-to-severe plaque psoriasis in paediatric patients $(>/=6$ to $<12$ years of age): efficacy, safety, pharmacokinetic and biomarker results from the open-label CADMUS Jr study. Br J Dermatol. 2020;183:664-672

7. Paller AS, Seyger MMB, Alejandro Magarinos G, et al. Efficacy and safety of ixekizumab in a phase III, randomized, double-blind, placebocontrolled study in paediatric patients with moderate-to-severe plaque psoriasis (IXORA-PEDS). Br J Dermatol. 2020;183:231-241.

8. Bruins FM, Bronckers I, Groenewoud HMM, et al. Association between quality of life and improvement in psoriasis severity and extent in pediatric patients. JAMA Dermatol. 2020;156:72-78.

9. Totri CR, Eichenfield LF, Logan K, et al. Prescribing practices for systemic agents in the treatment of severe pediatric atopic dermatitis in the US and Canada: the PeDRA TREAT survey. J Am Acad Dermatol. 2017;76:281-285

10. Paller AS, Siegfried EC, Vekeman F, et al. Treatment patterns of pediatric patients with atopic dermatitis: a claims data analysis. J Am Acad Dermatol. 2020;82:651-660.
11. Tsianakas A, Ständer S. Dupilumab: a milestone in the treatment of atopic dermatitis. The Lancet. 2016;10013:4-5

12. Simpson EL, Paller AS, Siegfried EC, et al. Efficacy and safety of dupilumab in adolescents with uncontrolled moderate to severe atopic dermatitis: a phase 3 randomized clinical trial. JAMA Dermatol. 2020;156:44-56.

13. Paller AS, Siegfried EC, Thaci D, et al. Efficacy and safety of dupilumab with concomitant topical corticosteroids in children 6 to 11 years old with severe atopic dermatitis: a randomized, double-blinded, placebo-controlled phase 3 trial. J Am Acad Dermatol. 2020;83:1282-1293.

14. Bagci IS, Ruzicka T. IL-31: a new key player in dermatology and beyond. J Allergy Clin Immunol. 2018;141:858-866.

15. Schwartz G, Paller AS. Targeted therapies for pediatric psoriasis. Semin Cutan Med Surg. 2018;37:167-172.

16. Dommasch ED, Kim SC, Lee MP, et al. Risk of serious infection in patients receiving systemic medications for the treatment of psoriasis. JAMA Dermatol. 2019;155:1142-1152.

17. Reich K, Blauvelt A, Armstrong A, et al. Secukinumab, a fully human anti-interleukin-17A monoclonal antibody, exhibits minimal immunogenicity in patients with moderate-to-severe plaque psoriasis. $\mathrm{Br} \mathrm{J}$ Dermatol. 2017;176:752-758.

18. Bagel J, Lebwohl M, Israel RJ, et al. Immunogenicity and skin clearance recapture in clinical studies of brodalumab. J Am Acad Dermatol. 2020;82:344-351.

19. Zhu Y, Marini JC, Song M, et al. Immunogenicity of guselkumab is not clinically relevant in patients with moderate-to-severe plaque psoriasis. I Invest Dermatol. 2019;139:1830.e6-1834.e6.

20. Langley RG, Kasichayanula S, Trivedi M, et al. Pharmacokinetics, immunogenicity, and efficacy of etanercept in pediatric patients with moderate to severe plaque psoriasis. J Clin Pharmacol. 2018; 58:340-346

21. Paller AS, Siegfried EC, Pariser DM, et al. Long-term safety and efficacy of etanercept in children and adolescents with plaque psoriasis. J Am Acad Dermatol. 2016;74:280-287.e1-3.

22. Papp K, Gottlieb AB, Naldi L, et al. Safety surveillance for ustekinumab and other psoriasis treatments from the Psoriasis Longitudinal Assessment and Registry (PSOLAR). J Drugs Dermatol. 2015;14:706-714.

23. Targeted immunomodulators for the treatment of moderateto-severe plaque psoriasis: effectiveness and value. Institute for Clinical and Economic Review website. https://icer-review.org /wp-content/uploads/2017/11/ICER_Psoriasis_Update_Draft _Report_04272018.pdf. Published December 2, 2016. Accessed October 26, 2020.

24. Dupilumab and crisaborole for atopic dermatitis: effectiveness and value. Institute for Clinical and Economic Review website. https://icer-review.org/wp-content/uploads/2016/10/MWCEPAC _ATOPIC_EVIDENCE_REPORT_051217.pdf. Published May 12, 2017. Accessed October 26, 2020.

25. Siegfried EC, Igelman S, Jaworski JC, et al. Use of dupilumab in pediatric atopic dermatitis: access, dosing, and implications for managing severe atopic dermatitis. Pediatr Dermatol. 2019;36:172-176.

26. Paller AS, Siegfried EC, Langley RG, et al. Etanercept treatment for children and adolescents with plaque psoriasis. $N$ Engl $\mathrm{J} \mathrm{Med}$. 2008;358:241-251.

27. Reich A. Secukinumab is highly efficacious and has a favorable safety profile in pediatric patients with moderate-to-severe plaque psoriasis. Presented at: AAD Virtual Meeting Experience; June 12-14, 2020. 


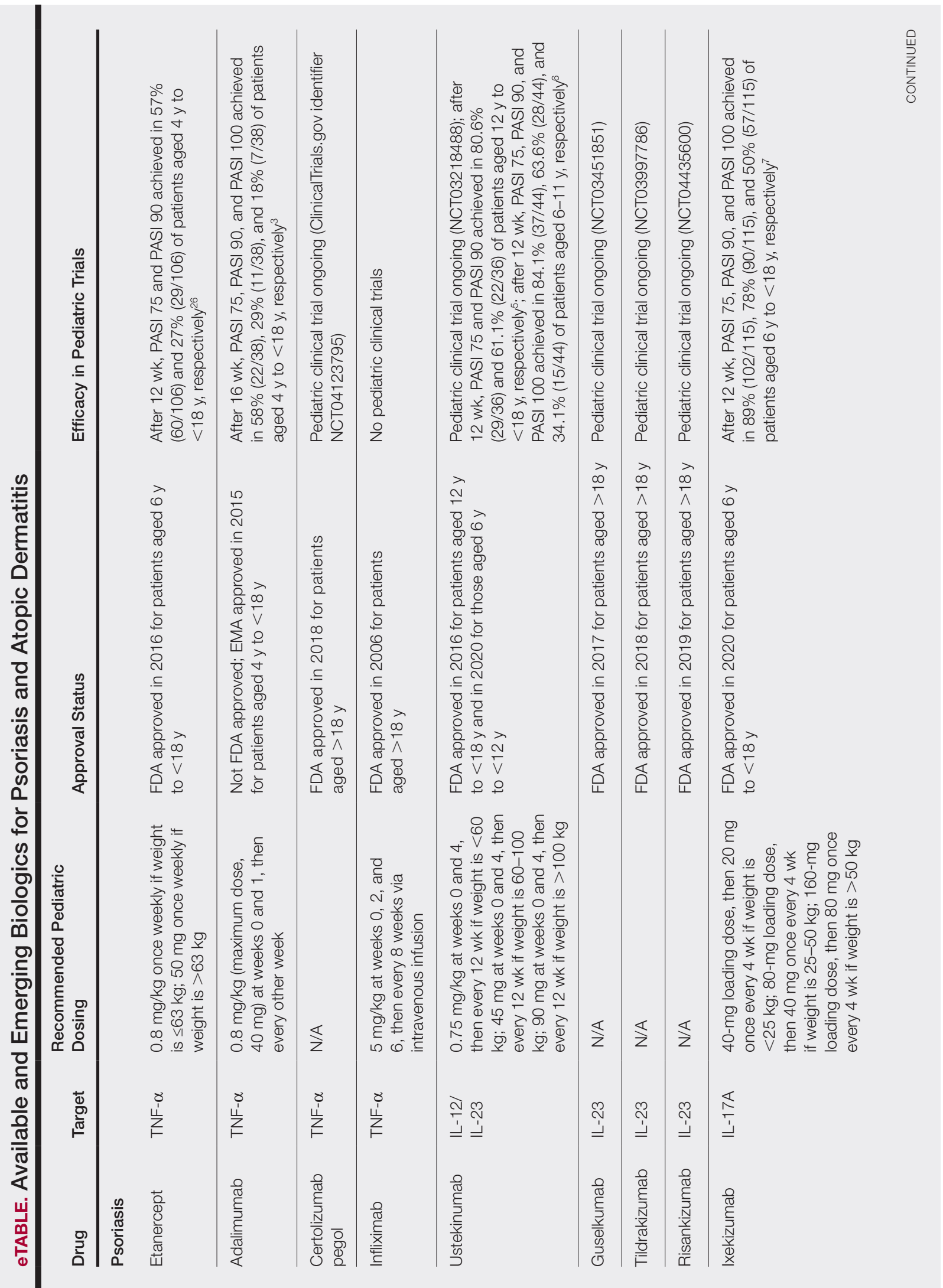




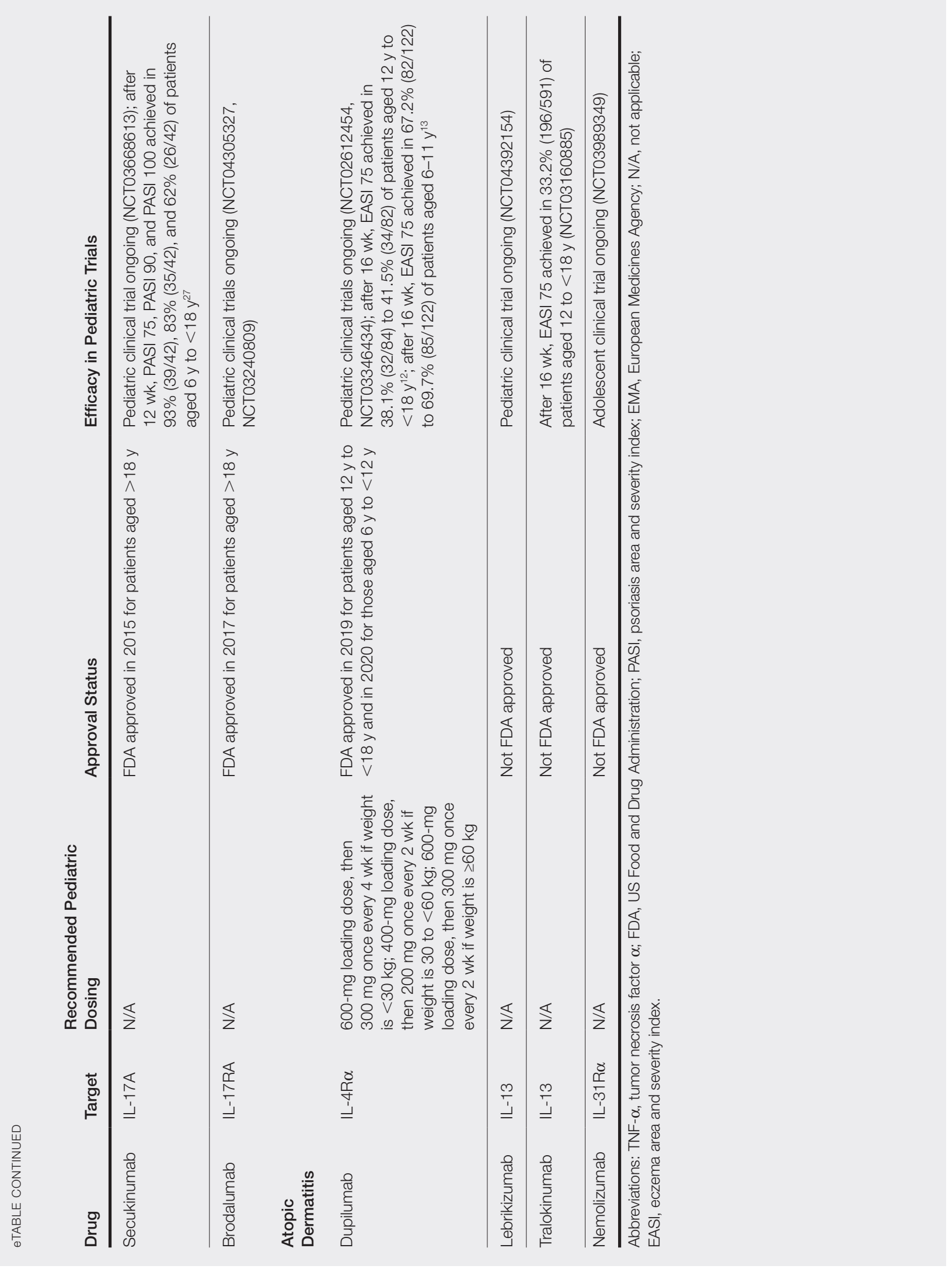

